\title{
The profiles of biopsy-proven renal tubulointerstitial lesions in patients with glomerular disease
}

\author{
Jin Dong ${ }^{1 \#}$, Yanqin Li $^{1 \#}$, Shuling Yue ${ }^{2 \#}$, Xiaoting Liu ${ }^{2}$, Long Wang ${ }^{1}$, Mengqi Xiong ${ }^{1}$, Guobao Wang ${ }^{1}$, \\ Sheng $\mathrm{Nie}^{1}$, Xin $\mathrm{Xu}^{1}$ \\ ${ }^{1}$ National Clinical Research Center for Kidney Disease, State Key Laboratory of Organ Failure Research, Nanfang Hospital, Southern Medical \\ University, Guangzhou, China; ${ }^{2}$ Department of Pathology, King Medical Diagnostics Center, Guangzhou, China \\ Contributions: (I) Conception and design: G Wang, S Nie, X Xu; (II) Administrative support: S Nie, X Xu; (III) Provision of study materials or \\ patients: L Wang, S Yue, X Liu, M Xiong; (IV) Collection and assembly of data: X Xu, J Dong, Y Li; (V) Data analysis and interpretation: S Nie, X \\ $\mathrm{Xu}$, J Dong; (VI) Manuscript writing: All authors; (VII) Final approval of manuscript: All authors. \\ \#These authors contributed equally to this work. \\ Correspondence to: Xin Xu; Sheng Nie. Renal Division, Nanfang Hospital, Southern Medical University, 1838 North Guangzhou Avenue, Guangzhou, \\ China. Email: xux007@163.com or niesheng0202@126.com.
}

Background: Renal tubules and interstitium are vulnerable to injury and play a central role in the progression of various chronic kidney diseases (CKDs). However, high quality epidemiologic study on the profiles of biopsy-proven tubulointerstitial lesions (TILs) is extremely limited.

Methods: We conducted a retrospective renal biopsy series including 62,569 native biopsies at 1,211 hospitals across China from 2015 to 2017 . The TILs, including the shedding of tube epithelial, renal tubular atrophy, renal interstitial fibrosis, edema and inflammatory infiltration, were identified from the pathological report. We analyzed the severity and chronicity of TILs stratified by gender, age groups, biopsy indications, and concurrent glomerular diseases. We also examined the correlation between TIL and glomerulosclerosis.

Results: Of 56,880 patients with biopsy-proven glomerular disease, 79.5\% had TILs. Renal interstitial inflammatory infiltration was the most common type of TIL (77.7\%), followed by renal tubular atrophy $(56.0 \%)$ and renal interstitial fibrosis $(32.8 \%)$. Severe and chronic TILs were more common in adults than in children. The three glomerular diseases with the highest proportion of moderate-to-severe and chronic TIL were diabetic nephropathy, immunoglobulin A (IgA) nephropathy and focal segmental glomerulosclerosis. The severity of TILs was moderately correlated with glomerulosclerosis score ( $\mathrm{r}=0.51)$. Moderate-to-severe and chronic TIL were more common in southern China. After adjusting for age, sex, hospital level, region, biopsy indication and type of concurrent glomerular diseases, adults with renal arteriole injury had a six-fold higher risk of moderate-to-severe TIL [odds ratio (OR), 7.12; 95\% confidence interval (CI), 6.42 to 7.91 ] and a three-fold higher risk of chronic TIL (OR, 4.58; 95\% CI, 4.37 to 4.79).

Conclusions: TILs were common in patients with biopsy-proven glomerular disease. The type and severity of TILs varied with age, region and concurrent glomerular diseases. Renal arteriole injury and glomerulosclerosis was associated with a significantly increased risk of TIL.

Keywords: Clinical epidemiology; kidney tubule; pathology injury; glomerulosclerosis; renal arteriole injury

Submitted Feb 18, 2020. Accepted for publication Jul 27, 2020.

doi: $10.21037 /$ atm-20-1669

View this article at: http://dx.doi.org/10.21037/atm-20-1669 


\section{Introduction}

Renal tubules and interstitium are the major components of kidney and susceptible to a variety of injures including hypoxia, proteinuria, systemic disorders, and nephrotoxic drugs. The glomerulus has long been thought the culprit for the progression of chronic kidney disease (CKD), while the renal tubules and interstitium are the victim of injury (1). In recent years, increasing evidence has demonstrated that renal function decline correlates better with tubulointerstitial lesions (TILs) than glomerular injury (2-4). It has been reported that tubular atrophy and fibrosis are better predictors of CKD progression than glomerular pathologic lesions. In patients with focal segmental glomerulosclerosis, the best clinical marker for disease progression is tubulointerstitial inflammation $(5,6)$. Similarly, in patients with immunoglobulin A (IgA) nephropathy, only tubulointerstitial injury was associated with the rate of estimated glomerular filtration rate (eGFR) loss over a follow-up period extending to 35 years (7). Crosstalk between tubulointerstitium and glomerulus may play a pivotal role in the prognosis of patients with primary glomerular lesions (8).

Most epidemiologic studies on renal biopsy have focused on the spectrum of glomerular diseases $(9,10)$. While a few studies focused on isolated tubulointerstitial disease such as acute or chronic tubulointerstitial nephritis (11-14), large and high-quality studies on the profiles of biopsy-proven TILs in patients with glomerular diseases are rather scarce.

In this study, we analyzed the profile of TILs in a renal biopsy series including 62,569 patients from 1,211 hospitals across China and evaluated their correlation with clinical indications and concurrent glomerular diseases. We present the following article in accordance with the STROBE reporting checklist (available at http://dx.doi.org/10.21037/ atm-20-1669).

\section{Methods}

\section{Study population and data collection}

We enrolled a total of 62,569 native renal biopsies from 1,211 hospitals across China from 2015 to 2017. All renal histologic specimens were processed and diagnosed at the Kingmed Pathology Center. The specimens were regularly stained with hematoxylin-eosin, periodic acidSchiff, periodic acid-silver Metheramine and Masson, and tested for $\operatorname{IgA}, \operatorname{IgG}, \operatorname{IgM}, \mathrm{C} 3, \mathrm{C} 4, \mathrm{C} 1 \mathrm{q}$ and $\mathrm{k} / \lambda$-light chains by immunofluorescence assays. Electron microscopic examination was also performed in $86.7 \%$ of the samples. The data collected included patients' demographic information, clinical syndrome or diagnosis at biopsy, date and hospital performing the biopsy, histologic diagnosis, detailed histological reports from light microscopy, electron microscopy, and immunofluorescence assays. The Medical Ethics Committee of Nanfang Hospital, Southern Medical University approved the study protocol. The study was conducted in accordance with the Declaration of Helsinki (as revised in 2013). The study was approved by The Medical Ethics Committee of Nanfang Hospital, Southern Medical University (No.: NFEC-2015-073) and individual consent for this retrospective analysis was waived.

\section{Identification of TILs}

Trained nephrologists reviewed the histological reports to identify TILs including both renal tubular lesions and renal interstitial lesions, as well as intrarenal arteriolar lesions. Renal tubular injuries included shedding of tubular epithelium and renal tubular atrophy. Renal interstitial injuries included edema, fibrosis, and infiltration of inflammatory cells. Intrarenal arteriolar lesions included arteriolar hyaline, arterial fibrotic intimal thickening, stenosis of the lumen and necrosis of intrarenal arterioles.

We categorized the severity of TILs based on the degree of spread out of inflammation, fibrosis, and atrophy. We defined focal or multifocal legion ( $<25 \%$ by area), flaky $(25-50 \%)$, and diffuse $(>50 \%)$ lesions as mild, moderate, and severe, respectively, with a corresponding score of 1 to 3 . Those without TILs were assigned a score of 0 . We defined chronic TILs as the existence of any tubular atrophy or fibrosis.

\section{Calculation of the glomerulosclerosis score}

The glomerulosclerosis score was calculated according to the percentage of glomeruli with global or segmental glomerulosclerosis (15). Samples with $<10 \%, 10-25 \%$, $26-50 \%$, and $>50 \%$ of glomeruli with global or segmental glomerulosclerosis were assigned a score from 0 to 3 , respectively.

\section{Classification of biopsy indications}

The indications for kidney biopsy were classified into one of the following categories in order (from high to low) based on the clinical diagnosis at biopsy: nephrotic syndrome 
(NS) with acute kidney injury (AKI), NS, AKI, progressive CKD defined as CKD stage 3-5, proteinuria without NS (PU), and isolated hematuria (HU). For cases that met the definitions of multiple categories, they were assigned to the class with the highest order.

\section{Statistical analyses}

We excluded the biopsy cases that had less than five glomeruli under light microscope, without histological diagnosis or glomerular disease, and the repeated biopsy cases. We summarized continuous variables by means \pm standard deviation (SD) or median (interquartile range) and categorical variables by count (percentage). We calculated the Spearman's correlation coefficient between the severity of TILs and the glomerulosclerosis score in the total samples as well as stratified by major glomerulopathies. We examined the association between the intrarenal arteriolar lesions and the severity of TILs in children and adults, respectively, using a logistic regression model with adjustment for age, sex, hospital categories, geologic region, biopsy indication and concurrent glomerular diseases. A natural spline term was used for the adjustment of age in the regression model.

A two-sided $\mathrm{P}$ value $<0.05$ was considered statistically significant. All statistical analyses were performed using $\mathrm{R}$ version 3.5.0 (16).

\section{Results}

\section{Study population}

We selected from biopsy series a total of 58,232 independent cases that had 5 or more glomeruli under the microscope with definite pathological diagnosis, including 340 with a combination of two different glomerulopathies, 56,880 with a single glomerulopathy, and 1,012 with isolated tubulointerstitial diseases. The profile of isolated tubulointerstitial diseases was listed in Table S1. We subsequently analyzed TILs in the cases that had a single biopsy-proven glomerular disease (Figure 1). Because the profiles of glomerulopathy differed significantly between children and adults, we analyzed TILs in children and adults separately.

The demographic and clinical characteristics of the biopsy cases analyzed, including 4,274 children and 52,606 adults, are presented in Table 1 . The study population was relatively young with an average age of 42 years and split equally in gender. Nearly $80 \%$ of the biopsy were performed in the tertiary class A hospitals in China. NS and proteinuria were the most common indications for renal biopsy in both children and adults. The profiles of glomerulopathy differed significantly between children and adults. The most common glomerular diseases in children were minimal change disease, Henoch-Schonlein purpura nephritis and IgA nephropathy, compared to membranous nephropathy, IgA nephropathy, and minimal change disease in adults.

\section{Profiles of TILs in patients with glomerular disease}

In our study population, TILs were present in $45.9 \%$ and $82.2 \%$ of the biopsy cases in children and adults, respectively (Table 2). The three most common TILs in children were interstitial inflammatory infiltration, shedding of tubular epithelium, and tubular atrophy, respectively. In adults, interstitial inflammatory infiltration, tubular atrophy and interstitial fibrosis were the most prevalent. Most of TILs were chronic lesions, and accompanied by intrarenal arteriolar lesions that were present in $13.3 \%$ of children and $66 \%$ of adults. The prevalence of chronic TILs and intrarenal arteriole lesions varied with biopsy indication and type of glomerulopathy (Figure 2). These lesions were much more common in adults than in children across the whole spectrum of biopsy indications and glomerulopathies, except for diabetic nephropathy, in which almost all cases $(>98 \%)$ have chronic TILs and intrarenal arteriole. Detailed profiles of TILs stratified by type of glomerulopathy were summarized in Table S2. Patients with AKI or progressive CKD were more likely to have chronic TILs as well as intrarenal arteriolar lesions in both children and adults.

\section{Risk factors of moderate-to-severe TILs}

In both children and adults, moderate-to-severe TILs were present in about $4 \%$ and $15 \%$ of cases with and without intrarenal arteriolar lesions, respectively (Table 3). After adjusting for other covariates, intrarenal arteriolar lesions were associated with a 7 -fold higher odds of moderate-tosevere TILs in both children [odds ratio (OR) 7.49; $95 \%$ confidence interval (CI), 5.23 to 10.74] and adults (OR 7.08; $95 \%$ CI, 6.38 to 7.87). As expected, the prevalence of moderate-to-severe TILs was much higher in patients with AKI or progressive CKD than those with other biopsy indications. Moderate-to-severe TILs was rare $(<1 \%)$ in patients with minimal change disease, but very common 


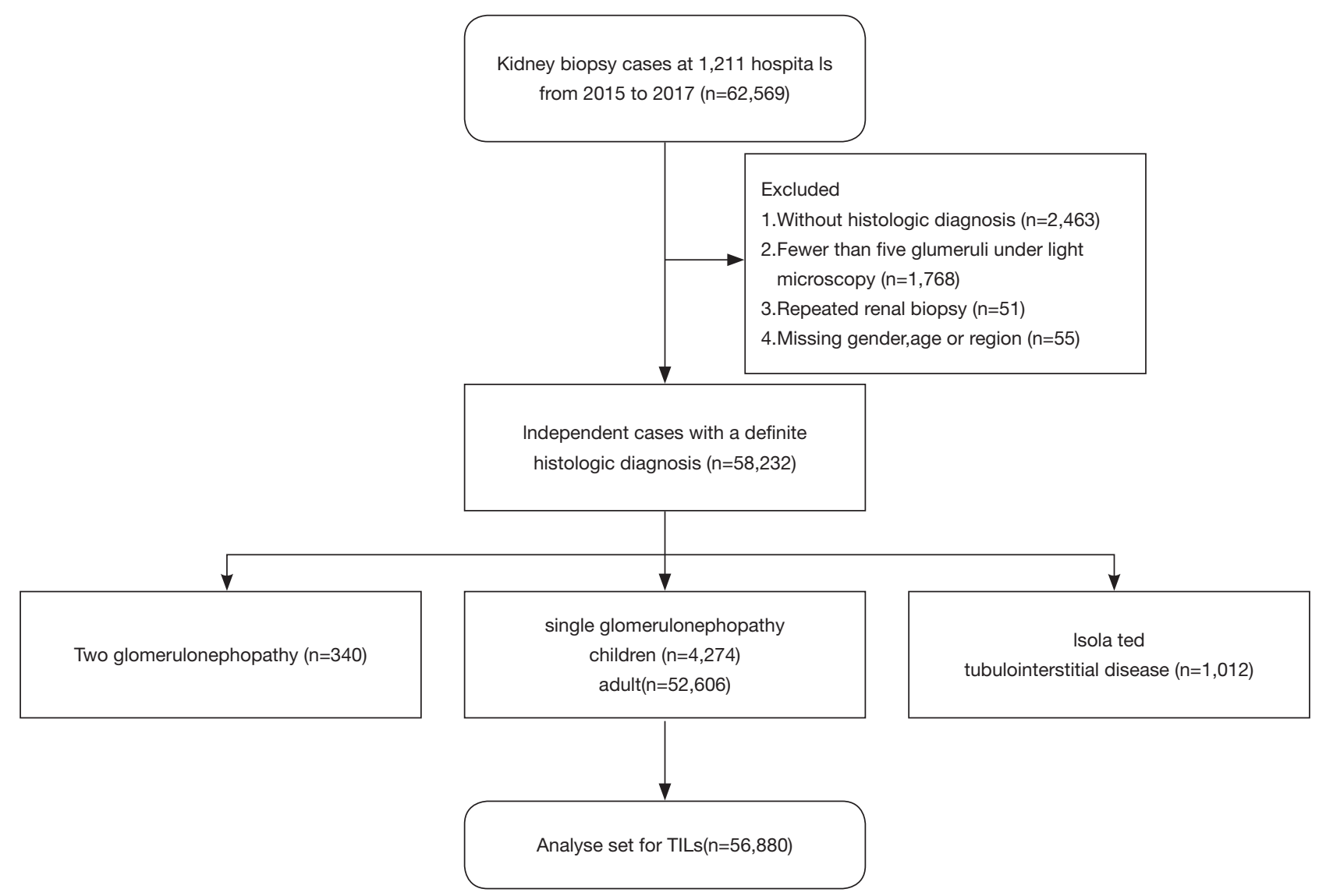

Figure 1 The flow chart of case selection.

(73.9\%) in those with diabetic nephropathy. Demographic risk factors of moderate-to-severe TILs included females in children, and males and Southerners in adult. The severity of TILs was also significantly correlated with glomerulosclerosis ( $\mathrm{r}=0.51$; 95\% CI, 0.50-0.52, Table S3), and the highest correlation observed in patients with IgA nephropathy $(\mathrm{r}=0.59$, Table S4).

\section{Risk factors of chronic TILs}

Chronic TILs were present in $20.6 \%$ of children and $66 \%$ of adults, most frequently found in patients with IgA nephropathy (82.3\%) and diabetic nephropathy (98.3\%). In both adult and children, intrarenal arteriolar lesions, AKI, and progressive CKD were associated with a significantly increased risk of chronic TILs after adjusting for other covariates (Table 4).

\section{Discussion}

To the best of our knowledge, this is the first nationwide study to describe the profiles of biopsy-proven TILs among patients with glomerular disease. Although the biopsy samples in our study came from 1,211 hospitals across China, all the samples were processed and diagnosed histologically at a single pathological laboratory using unified criteria, which minimized the heterogeneity in histological diagnosis. In our study, TILs were more common in adults than in children. Patients with intrarenal arteriolar lesions, glomerulosclerosis, AKI, progressive $\mathrm{CKD}$, or diabetic nephropathy had a significantly increased risk of moderate-to-severe and chronic TILs. 
Table 1 Characteristics of the biopsy series in China

\begin{tabular}{|c|c|c|c|}
\hline Characteristics & All patients $(n=56,880)$ & Children $(n=4,274)$ & Adults $(n=52,606)$ \\
\hline \multicolumn{4}{|l|}{ Gender, N (\%) } \\
\hline Male & $30,512(53.6)$ & $2,665(62.4)$ & 27,847 (52.9) \\
\hline Female & $26,368(46.4)$ & $1,609(37.6)$ & $24,759(47.1)$ \\
\hline NS & $26,366(46.4)$ & $1,836(43.0)$ & $24,530(46.6)$ \\
\hline Proteinuria & 22,196 (39.0) & $1,818(42.5)$ & $20,378(38.7)$ \\
\hline Progressive $C K D^{a}$ & 3,354 (5.9) & $62(1.5)$ & $3,292(6.3)$ \\
\hline Hematuria & $616(1.1)$ & $225(5.3)$ & $391(0.7)$ \\
\hline Unknown & $3,464(6.1)$ & $258(6.0)$ & $3,206(6.1)$ \\
\hline \multicolumn{4}{|l|}{ Hospital level, N (\%) } \\
\hline Tertiary class $A^{b}$ & 44,505 (78.2) & $3,762(88.0)$ & $40,743(77.4)$ \\
\hline Others & $12,375(21.8)$ & $512(12.0)$ & $11,863(22.6)$ \\
\hline \multicolumn{4}{|l|}{ Region, N (\%) } \\
\hline Central & $5,728(10.1)$ & $363(8.5)$ & $5,365(10.2)$ \\
\hline East & $18,975(33.4)$ & $834(19.5)$ & $18,141(34.5)$ \\
\hline North & $9,519(16.7)$ & $323(7.6)$ & $9,196(17.5)$ \\
\hline MCD & 7,353 (12.9) & $1,221(28.6)$ & $6,132(11.7)$ \\
\hline MsPGN & $2,825(5.0)$ & $183(4.3)$ & $2,642(5.0)$ \\
\hline FSGS & $2,526(4.4)$ & $199(4.7)$ & $2,327(4.4)$ \\
\hline LN & $3,290(5.8)$ & $341(8.0)$ & $2,949(5.6)$ \\
\hline HSPN & $1,931(3.4)$ & $922(21.6)$ & $1,009(1.9)$ \\
\hline DN & $1,692(3.0)$ & $1(0.0)$ & $1,691(3.2)$ \\
\hline Others & 3,940 (6.9) & $360(8.4)$ & $3,580(6.8)$ \\
\hline
\end{tabular}

${ }^{a}$, define as CKD stage $3-5 ;{ }^{b}$, according to the classification of Chinese hospitals. NS, nephrotic syndrome; CKD, chronic kidney disease; AKI, acute kidney injury; MN, membranous nephropathy; IgAN, IgA nephropathy; MCD, minimal change disease; FSGS, focal segmental glomerulosclerosis; MsPGN, mesangial proliferative glomerulonephritis; DN, diabetic nephropathy; LN, lupus Nephropathy; HSPN, henoch-schonlein purpura nephritis. 
Table 2 TILs and intrarenal arteriolar lesions

\begin{tabular}{llll}
\hline Various TILs & All patients, N=56,880 (\%) & Children, N=4,274 (\%) & Adults, N=52,606 (\%) \\
\hline Renal tubular lesions & $40,422(71.1)$ & $1,420(33.2)$ & $39,002(74.1)$ \\
$\quad$ Shedding of tubular epithelial & $12,489(22.0)$ & $922(21.6)$ & $11,567(22.0)$ \\
Renal tubular atrophy & $31,843(56.0)$ & $584(13.7)$ & $31,259(59.4)$ \\
Renal interstitial lesions & $44,237(77.8)$ & $1,832(42.9)$ & $42,405(80.6)$ \\
Tubulointerstitial edema & $9,399(16.5)$ & $561(13.1)$ & $8,838(16.8)$ \\
Renal interstitial fibrosis & $18,681(32.8)$ & $474(11.1)$ & $18,207(34.6)$ \\
Inflammatory cell infiltration & $44,203(77.7)$ & $1,830(42.8)$ & $42,373(80.5)$ \\
Intrarenal arteriolar lesions & $35,328(62.1)$ & $569(13.3)$ & $34,759(66.1)$ \\
Arteriolar hyaline & $2,384(4.2)$ & $9(0.2)$ & $2,375(4.5)$ \\
Arterial fibrotic intimal thickening & $34,666(60.9)$ & $526(12.3)$ & $34,140(64.9)$ \\
$\quad$ Stenosis of the lumen & $35,268(62.0)$ & $566(13.2)$ & $34,702(66.0)$ \\
Necrosis of intrarenal arteriole & $45(0.1)$ & $1(0.0)$ & $44(0.1)$
\end{tabular}

TILs, tubulointerstitial lesions.

In our study, male was associated with a slightly increased risk of moderate-to-severe TILs in adults and a decreased risk in children after adjusting for other covariates. The exact mechanisms for these associations are not clear, and it may be caused by unadjusted confounders. For example, adult males are more likely to have hypertension and diabetes and to be exposed to nephrotoxic drugs (17). Such information was not available and hence not included in our association analyses.

The intrarenal arteriolar lesion and glomerulosclerosis are significant contributors of TILs in our study. A previous study reported that the proportions of tubular atrophy, interstitial cell infiltration, and interstitial fibrosis were significantly higher in patients with intrarenal arteriolar lesions than that in the patients without arteriolar lesions (18). Since the peritubular capillaries represent the sole blood supply to the tubules, glomerular scarring that damages the upstream capillary bed could have downstream consequences. Bohle et al. (19) found a significant inverse relationship between post-glomerular capillary area and serum creatinine. Obliteration of the peritubular capillary network could lead to reduce blood flow, which results in tubule hypoxia and tubular epithelial cell death and tubular atrophy (20-22). Meanwhile, tubulointerstitial and vessel damage increases the vascular resistance, which leads to drop in the glomerular filtration rate and eventually enters a vicious circle (23). Consistent with previous reports (24-26), the grade of glomerulosclerosis was significantly associated with the severity of TILs in our study, especially in patients with IgA nephropathy.

In our study, the highest prevalence of moderateto-severe TIL was observed in patients with diabetic nephropathy, followed by IgA nephropathy. It has been reported that activation of renal local complement in patients with diabetic nephropathy contributes to kidney damage, especially tubular interstitial damage (27). Meanwhile, the arterial lesion is common in patients with diabetes which might contribute to the development of TIL. For IgA nephropathy, our results slightly differ from the findings of the previous work from China, in which $38.3 \%$ of patients with IgA nephropathy had moderate to severe TIL (28).

It is noteworthy that patients from southern China, mostly Guangdong province, had a $57 \%$ higher risk of moderate-to-severe TILs than that from other regions. According to the National Adverse Drug Reaction (ADR) Monitoring System in China, 33.7\% of ADR in Guangdong province were potentially caused by inappropriate medication (29), which was the highest among all provinces. In Guangdong, use of Chinese herbal medicine is very common, and the incidence of new or serious ADRs of traditional Chinese herbal injections is significantly higher than the national average (30). We speculate that higher exposure to potentially nephrotoxic drugs may partly 


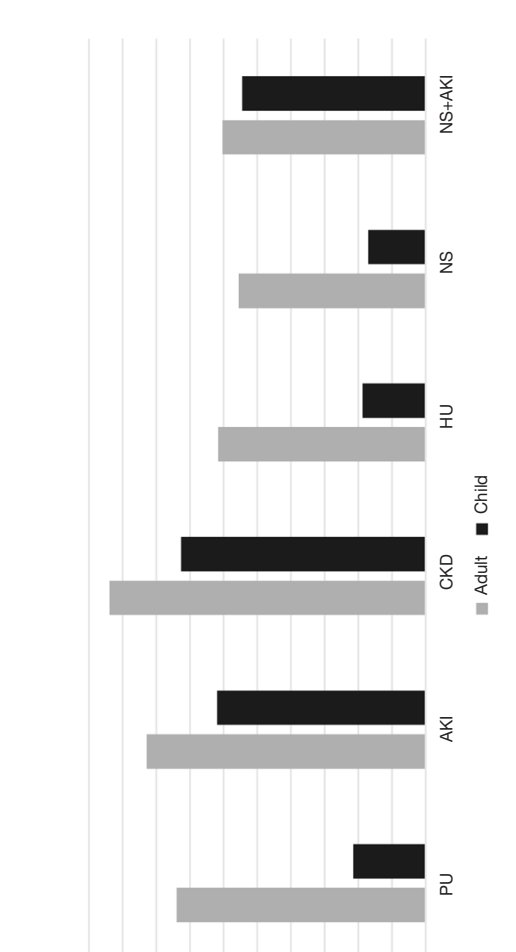

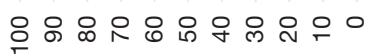

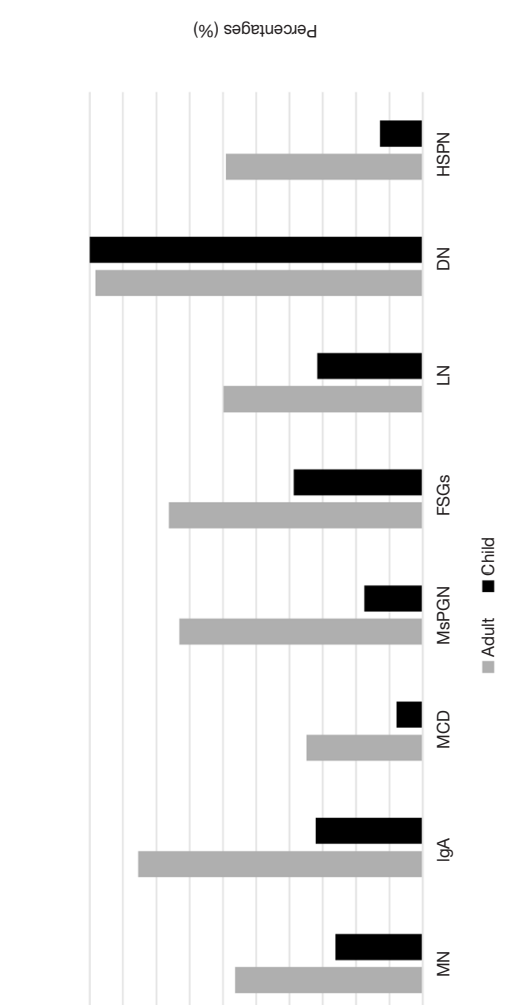

응ㅇㅇㅇㅇㅇㅇㅇㅇㅇㅇㅇㅇ응

$\varangle$

(\%) sә6етuәраә

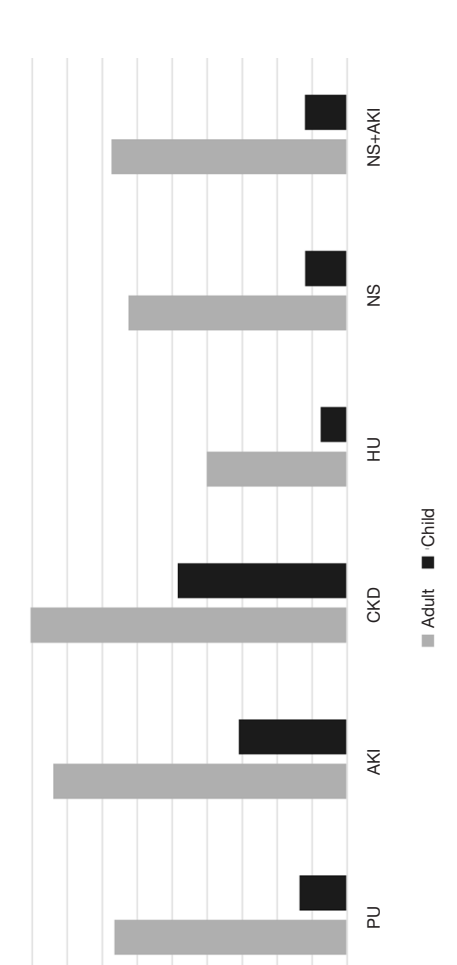

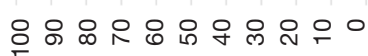

(\%) sә6еңиәэәд

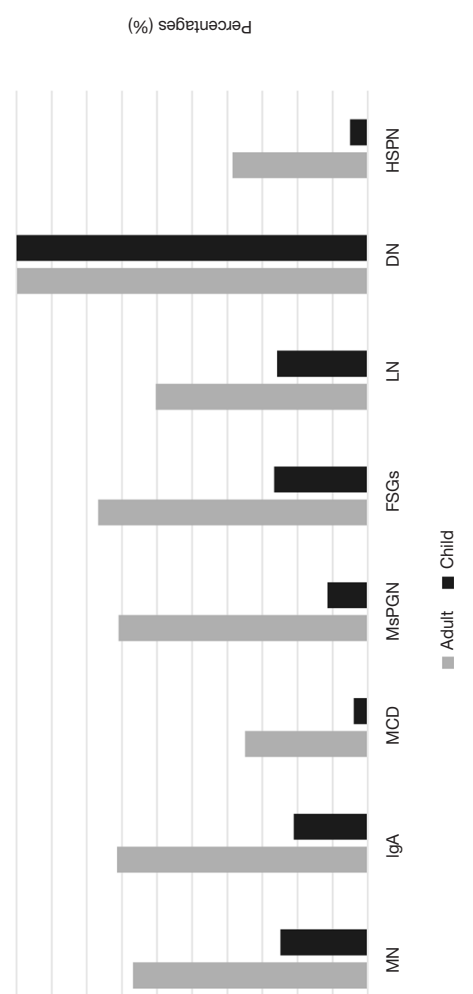

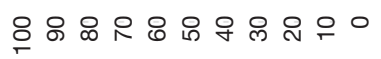

$\infty$

(\%) sәбеңиәэәд

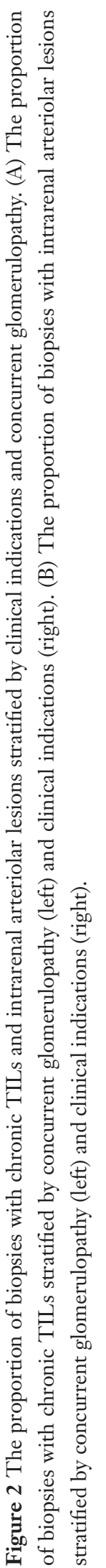




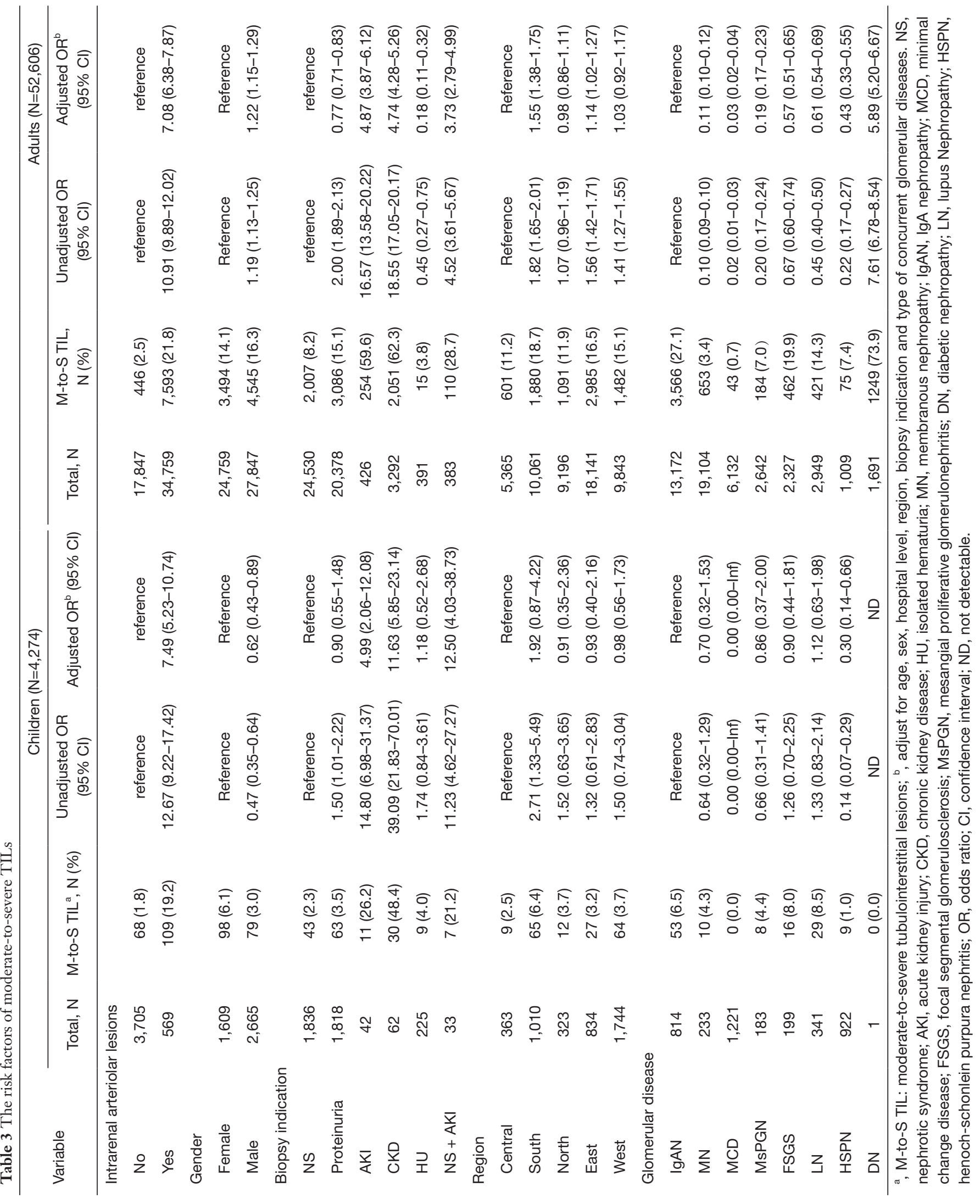


Table 4 The risk factors of chronic TILs

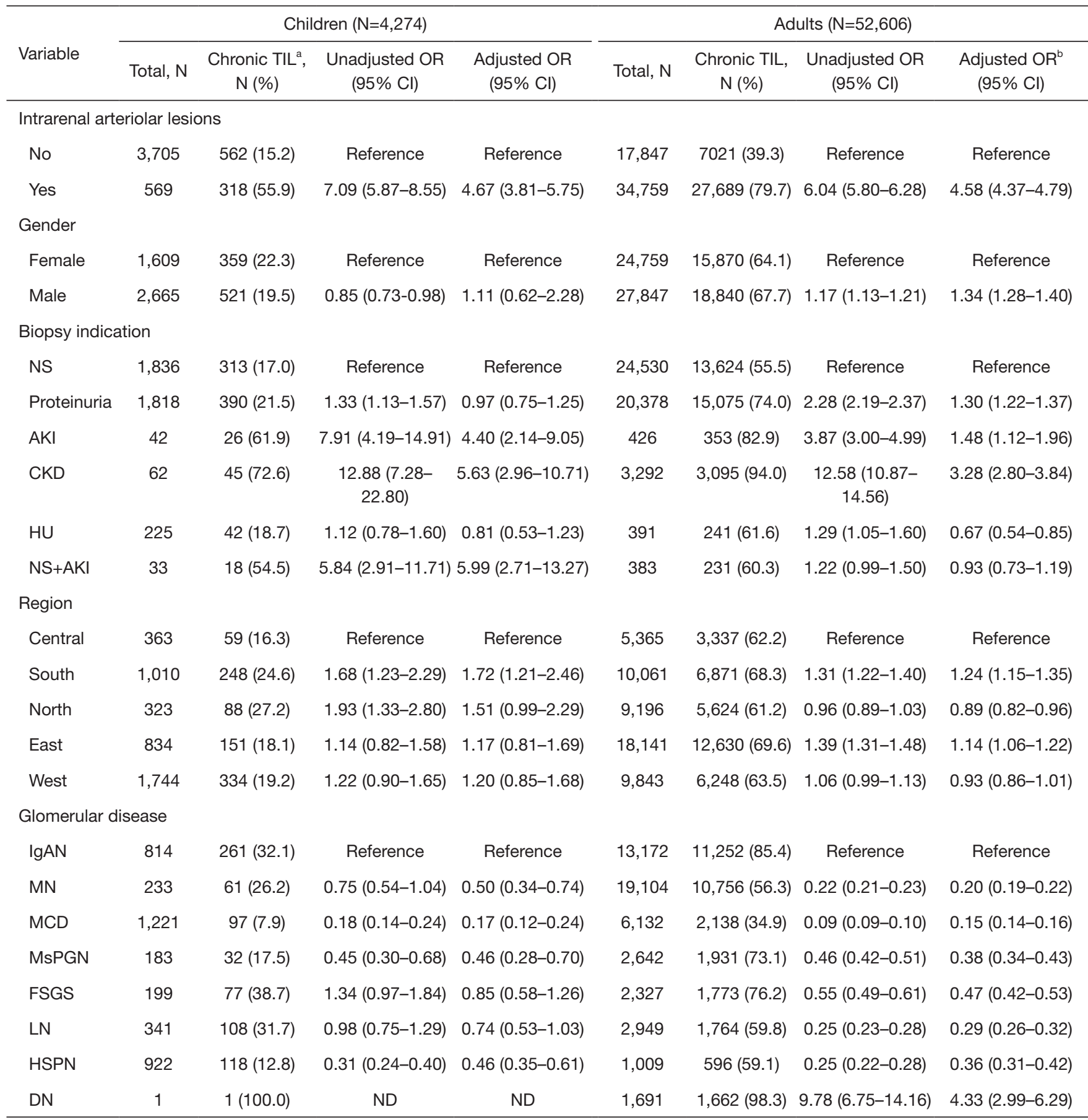

a, Chronic TIL: existence of any tubular atrophy or fibrosis; ${ }^{b}$, adjust for age, sex, hospital level, region, biopsy indication and type of concurrent glomerular diseases. TILs, tubulointerstitial lesions; NS, nephrotic syndrome; AKI, acute kidney injury; CKD, chronic kidney disease; HU, isolated hematuria; MN, membranous nephropathy; IgAN, IgA nephropathy; MCD, minimal change disease; FSGS, focal segmental glomerulosclerosis; MsPGN, mesangial proliferative glomerulonephritis; DN, diabetic nephropathy; LN, lupus Nephropathy; HSPN, henoch-schonlein purpura nephritis; OR, odds ratio; $\mathrm{Cl}$, confidence interval; ND, not detectable. 
contribute to the observed increased risk of moderate-tosevere TIL in patients from southern China (31).

Our study has several limitations. First, we do not have detailed clinical data of the patients such as laboratory indices, comorbidities and medications which may confound our association analyses of moderate-to-severe TILs. However, biopsy indications such as NS, proteinuria and hematuria may serve as a semi-quantitative surrogate for the severity of disease. Second, because our study is crosssectional we are not able to evaluate the prognosis of TILs. Third, like any association studies, we are not able to make causal inference between TILs and the risk factors. Fourth, the present study only included Chinese population. Whether these results are generalizable to other ethnic population is unknown.

In conclusion, TIL is very frequent in patients with glomerular disease, and its profile varies greatly with age, biopsy indication, and concurrent glomerular disease. Renal arteriolar lesion is associated with a significantly increased risk of TIL. Given the pivotal role of TIL in the progression of kidney disease, more epidemiologic studies are needed to establish the long-term prognostic value of TIL in patients with glomerular disease.

\section{Acknowledgments}

Funding: This work was supported by the National Natural Science Foundation of China (Key Program) (81430016), the National Natural Science Foundation of China (81770683, 81900626 and 81970586), and the Major International (Regional) Joint Research Project (81620108003), Guangzhou Regenerative Medicine and Health-Guangdong Laboratory Research Grant (2018GZR0201003).

\section{Footnote}

Reporting Checklist: The authors have completed the STROBE reporting checklist. Available at http://dx.doi. org/10.21037/atm-20-1669

Data Sharing Statement: Available at http://dx.doi. org/10.21037/atm-20-1669

Conflicts of Interest: All authors have completed the ICMJE uniform disclosure form (available at http://dx.doi. org/10.21037/atm-20-1669). The authors have no conflicts of interest to declare.
Ethical Statement: The authors are accountable for all aspects of the work in ensuring that questions related to the accuracy or integrity of any part of the work are appropriately investigated and resolved. The study was conducted in accordance with the Declaration of Helsinki (as revised in 2013). The study was approved by The Medical Ethics Committee of Nanfang Hospital, Southern Medical University (No.: NFEC-2015-073) and individual consent for this retrospective analysis was waived.

Open Access Statement: This is an Open Access article distributed in accordance with the Creative Commons Attribution-NonCommercial-NoDerivs 4.0 International License (CC BY-NC-ND 4.0), which permits the noncommercial replication and distribution of the article with the strict proviso that no changes or edits are made and the original work is properly cited (including links to both the formal publication through the relevant DOI and the license). See: https://creativecommons.org/licenses/by-nc-nd/4.0/.

\section{References}

1. Liu BC, Tang TT, Lv LL, et al. Renal tubule injury: a driving force toward chronic kidney disease. Kidney Int 2018;93:568-79.

2. Risdon RA, Sloper JC, De Wardener HE. Relationship between renal function and histological changes found in renal-biopsy specimens from patients with persistent glomerular nephritis. Lancet 1968;2:363-6.

3. Bohle A, Christ H, Grund KE, et al. The role of the interstitium of the renal cortex in renal disease. Contrib Nephrol 1979;16:109-14.

4. Mackensen-Haen S, Bader R, Grund KE, et al. Correlations between renal cortical interstitial fibrosis, atrophy of the proximal tubules and impairment of the glomerular filtration rate. Clin Nephrol 1981;15:167-71.

5. Bonsib SM. Focal-segmental glomerulosclerosis. The relationship between tubular atrophy and segmental sclerosis. Am J Clin Pathol 1999;111:343-8.

6. Schainuck LI, Striker GE, Cutler RE, et al. Structuralfunctional correlations in renal disease. II. The correlations. Hum Pathol 1970;1:631-41.

7. Coppo R, D'Arrigo G, Tripepi G, et al. Is there longterm value of pathology scoring in immunoglobulin A nephropathy? A validation study of the Oxford Classification for IgA Nephropathy (VALIGA) update. Nephrol Dial Transplant 2020;35:1002-9.

8. Kriz W, Gretz N, Lemley KV. Progression of glomerular 
diseases: is the podocyte the culprit? Kidney Int 1998;54:687-97.

9. Xu X, Wang G, Chen N, et al. Long-Term Exposure to Air Pollution and Increased Risk of Membranous Nephropathy in China. J Am Soc Nephrol 2016;27:3739-46.

10. Nie S, He W, Huang T, et al. The Spectrum of BiopsyProven Glomerular Diseases among Children in China: A National, Cross-Sectional Survey. Clin J Am Soc Nephrol 2018;13:1047-54.

11. Muriithi AK, Leung N, Valeri AM, et al. Biopsy-proven acute interstitial nephritis, 1993-2011: a case series. Am J Kidney Dis 2014;64:558-66.

12. Goicoechea M, Rivera F, López-Gómez JM. Increased prevalence of acute tubulointerstitial nephritis. Nephrol Dial Transplant 2013;28:112-5.

13. Su T, Gu Y, Sun P, et al. Etiology and renal outcomes of acute tubulointerstitial nephritis: a single-center prospective cohort study in China. Nephrol Dial Transplant 2018;33:1180-8.

14. Wilson GJ, Kark AL, Francis LP, et al. The increasing rates of acute interstitial nephritis in Australia: a single centre case series. BMC Nephrol 2017;18:329.

15. Sethi S, D'Agati VD, Nast CC, et al. A proposal for standardized grading of chronic changes in native kidney biopsy specimens. Kidney Int 2017;91:787-9.

16. Schuenemeyer JH. Generalized Linear Models. Technometrics 1992;34:223-4.

17. Kramer A, Pippias M, Noordzij M, et al. The European Renal Association - European Dialysis and Transplant Association (ERA-EDTA) Registry Annual Report 2015: a summary. Clin Kidney J 2018;11:108-22.

18. Wu J, Chen X, Xie Y, et al. Characteristics and risk factors of intrarenal arterial lesions in patients with IgA nephropathy. Nephrol Dial Transplant 2005;20:719-27.

19. Bohle A, Müller GA, Wehrmann M, et al. Pathogenesis of chronic renal failure in the primary glomerulopathies, renal vasculopathies, and chronic interstitial nephritides. Kidney Int Suppl 1996;54:S2-9.

20. Matsumoto M, Tanaka T, Yamamoto T, et al. Hypoperfusion of peritubular capillaries induces chronic

Cite this article as: Dong J, Li Y, Yue S, Liu X, Wang L, Xiong $M$, Wang G, Nie S, Xu X. The profiles of biopsy-proven renal tubulointerstitial lesions in patients with glomerular disease. Ann Transl Med 2020;8(17):1066. doi: 10.21037/atm20-1669 hypoxia before progression of tubulointerstitial injury in a progressive model of rat glomerulonephritis. J Am Soc Nephrol 2004;15:1574-81.

21. Rosenberger C, Khamaisi M, Abassi Z, et al. Adaptation to hypoxia in the diabetic rat kidney. Kidney Int 2008;73:34-42.

22. Fine LG, Norman JT. Chronic hypoxia as a mechanism of progression of chronic kidney diseases: from hypothesis to novel therapeutics. Kidney Int 2008;74:867-72.

23. Okada H, Suzuki H, Konishi K, et al. Histological alterations in renal specimens as indicators of prognosis of IgA nephropathy. Clin Nephrol 1992;37:235-8.

24. Kriz W, LeHir M. Pathways to nephron loss starting from glomerular diseases-insights from animal models. Kidney Int 2005;67:404-19.

25. Healy E, Brady HR. Role of tubule epithelial cells in the pathogenesis of tubulointerstitial fibrosis induced by glomerular disease. Curr Opin Nephrol Hypertens 1998;7:525-30.

26. Masum MA, Ichii O, Elewa YHA, et al. Local CD34positive capillaries decrease in mouse models of kidney disease associating with the severity of glomerular and tubulointerstitial lesions. BMC Nephrol 2017;18:280.

27. Zheng JM, Ren XG, Jiang ZH, et al. Lectin-induced renal local complement activation is involved in tubular interstitial injury in diabetic nephropathy. Clin Chim Acta 2018;482:65-73.

28. Zhang Y, Chen X, Zhuang Y. Clinical analysis of tubulointerstitial lesions in IgA nephropathy. Zhonghua Nei Ke Za Zhi 2001;40:613-7.

29. Ma GY, Deng JX, Jiang J. Irrational drug usage in adverse drug reaction database of Guangdong. Pharmacy Today 2009. Available online: http://en.cnki.com.cn/Article_en/ CJFDTOTAL-YAXU200903006.htm

30. Li H, Deng J, Deng L, et al. Safety profile of traditional Chinese herbal injection: An analysis of a spontaneous reporting system in China. Pharmacoepidemiol Drug Saf 2019;28:1002-13.

31. Yang B, Xie Y, Guo M, et al. Nephrotoxicity and Chinese Herbal Medicine. Clin J Am Soc Nephrol 2018;13:1605-11. 
Table S1 The spectrum of isolated tubulointerstitial diseases

\begin{tabular}{ll}
\hline Disease & Cases \\
\hline Acute interstitial nephritis & 395 \\
Chronic interstitial nephritis & 304 \\
Acute tubular necrosis & 182 \\
Subacute interstitial nephritis & 86 \\
Multiple myeloma kidney injury & 19 \\
Sjogren's syndrome kidney injury & 9 \\
Renal interstitial infiltration by tumor cells & 6 \\
IgG4-related nephropathy & 6 \\
Gitelman syndrome & 1 \\
Chronic uric acid nephropathy & 1 \\
Subacute tubular necrosis & 1 \\
Renal cystic disease & 1 \\
Oxalate nephropathy & 1 \\
\hline
\end{tabular}

Table S2 The types of TILs stratified by glomerular disease

\begin{tabular}{|c|c|c|c|c|c|c|c|c|}
\hline Pathologic changes & $\mathrm{DN}, \mathrm{N}=1,692$ & $\lg A N, N=13,986$ & FSGS, $N=2,526$ & $\mathrm{MN}, \mathrm{N}=19,337$ & $\mathrm{MCD}, \mathrm{N}=7,353$ & MsPGN, N=2,825 & $L N, N=3,290$ & HSPN, $N=1,931$ \\
\hline Renal tubular lesions, $\mathrm{n}(\%)$ & $1,636(96.7)$ & $12,064(86.3)$ & $2,159(85.5)$ & $12,796(66.2)$ & $3,319(45.1)$ & $2,070(73.3)$ & $2,252(68.4)$ & $907(47.0)$ \\
\hline Shedding of tubular epithelial & $76(4.5)$ & $2,178(15.6)$ & $739(29.3)$ & $4,344(22.5)$ & $1,847(25.1)$ & $318(11.3)$ & $1,202(36.5)$ & $348(18.0)$ \\
\hline Renal tubular atrophy & $1,605(94.9)$ & $10,921(78.1)$ & $1,679(66.5)$ & $9,814(50.8)$ & $1,848(25.1)$ & $1,901(67.3)$ & $1,323(40.2)$ & $636(32.9)$ \\
\hline Renal interstitial lesions, $\mathrm{n}(\%)$ & $1,686(99.6)$ & $12,531(89.6)$ & $2,282(90.3)$ & $14,661(75.8)$ & $3,437(46.7)$ & $2,189(77.5)$ & $2800(85.1)$ & $1,098(56.9)$ \\
\hline Tubulointerstitial edema & $116(6.9)$ & $2,081(14.9)$ & $511(20.2)$ & $2,964(15.3)$ & $850(11.6)$ & $207(7.3)$ & $1,032(31.4)$ & $238(12.3)$ \\
\hline Renal interstitial fibrosis & $1,583(93.6)$ & $7,245(51.8)$ & $1,125(44.5)$ & $3,432(17.7)$ & $585(8.0)$ & $918(32.5)$ & $1,270(38.6)$ & $271(14.0)$ \\
\hline Inflammatory cell infiltration & $1,685(99.6)$ & $12,525(89.6)$ & $2,281(90.3)$ & $14,645(75.7)$ & $3,430(46.6)$ & $2,189(77.5)$ & $2,799(85.1)$ & $1,098(56.9)$ \\
\hline Intrarenal arteriolar lesions, $\mathrm{n}(\%)$ & $1,688(99.8)$ & $9,576(68.5)$ & $1,839(72.8)$ & $12,817(66.3)$ & $2,191(29.8)$ & $1,896(67.1)$ & $1,869(56.8)$ & $435(22.5)$ \\
\hline Arteriolar hyaline & $1,228(72.6)$ & $310(2.2)$ & $118(4.7)$ & $283(1.5)$ & $60(0.8)$ & $184(6.5)$ & $22(0.7)$ & $16(0.8)$ \\
\hline Arterial fibrotic intimal thickening & $1,683(99.5)$ & $9,457(67.6)$ & $1,819(72.0)$ & $12,527(64.8)$ & $2,084(28.3)$ & $1,870(66.2)$ & $1,828(55.6)$ & $416(21.5)$ \\
\hline Stenosis of the lumen & $1,685(99.6)$ & $9,563(68.4)$ & $1,835(72.6)$ & $12,802(66.2)$ & $2,177(29.6)$ & $1,894(67.0)$ & $1,868(56.8)$ & $433(22.4)$ \\
\hline Necrosis of intrarenal arteriole & $0(0.0)$ & $2(0.0)$ & $0(0.0)$ & $0(0.0)$ & $0(0.0)$ & $0(0.0)$ & $5(0.2)$ & $0(0.0)$ \\
\hline
\end{tabular}

a , Chronic TIL: as presence of any tubular atrophy or fibrosis. DN, diabetic nephropathy; IgAN, IgA nephropathy. 
Table S3 The TILs lesions stratified by glomerulosclerosis score

\begin{tabular}{lccr}
\hline \multirow{2}{*}{ Glomerulosclerosis score } & \multicolumn{3}{c}{ Severity of TILs } \\
\cline { 2 - 4 } & None & Mild & Moderate-to-severe \\
\hline 0 & $9,674(40.8)$ & $13,120(55.4)$ & $897(3.8)$ \\
1 & $2,539(10.6)$ & $18,887(79.1)$ & $2,441(10.2)$ \\
3 & $75(1.1)$ & $3735(57.3)$ & $2,712(41.6)$ \\
\hline
\end{tabular}

TILs, tubulointerstitial lesions.

Table S4 The correlation between the severity of TILs and glomerulosclerosis stratified by glomerulopathy

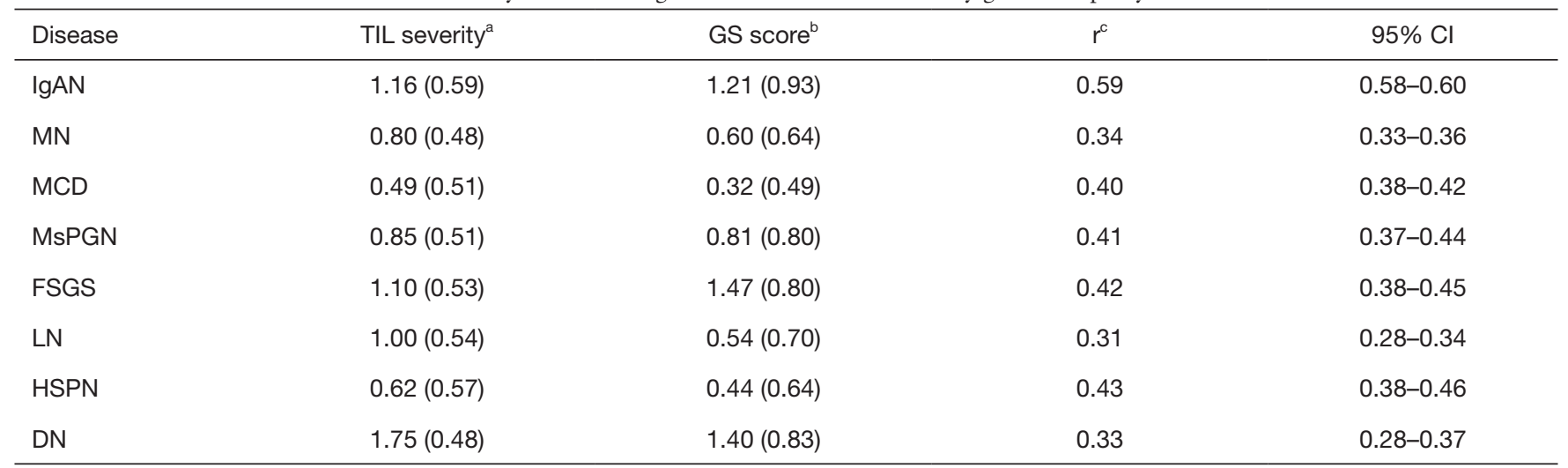

a , present as mean (SD); ${ }^{b}$, glomerulosclerosis score present as mean (SD); ${ }^{c}$, Spearman correlation coefficient. TILs, tubulointerstitial lesions; MN, membranous nephropathy; IgAN, IgA nephropathy; MCD, minimal change disease; FSGS, focal segmental glomerulosclerosis; MsPGN, mesangial proliferative glomerulonephritis; DN, diabetic nephropathy; LN, lupus Nephropathy; HSPN, henoch-schonlein purpura nephritis. 